\title{
SYMBOLS FOR TRACE CLASS HANKEL OPERATORS WITH GOOD ESTIMATES FOR NORMS
}

\author{
by F. F. BONSALL and D. WALSH
}

(Received 25 October, 1984)

Introduction. Peller $[4,5]$ has proved that a Hankel operator $S$ on the Hardy space $H^{2}$ is in the trace class if and only if $S=S_{\bar{h}}$ with $h$ analytic on the open unit disc $D$ and with its second derivative belonging to the Bergman space $L_{a}^{1}$. This theorem does not include an estimate for the trace class norm $\|S\|_{1}$ of the operator in terms of the symbol function. In fact it is clear that $\left\|h^{\prime \prime}\right\|_{L_{a}^{1}}$ cannot give an estimate for $\left\|S_{h}\right\|_{1}$ since the first two terms in the coefficient sequence of the Hankel operator have been removed by differentiation.

We give a slightly modified version of Peller's theorem which eliminates this difficulty and leads to a satisfactory estimate for $\|S\|_{1}$. The proof uses a modified version of the Coifman-Rochberg decomposition theorem for $L_{a}^{1},[3]$. As a corollary, we obtain a bounded projection of the trace class onto its Hankel operators, again with a good estimate of the norm. For other bounded projections with the same domain and range, see $[4]$.

Notation. Let $L^{p}=L^{p}(\partial D)$ with normalized Lebesgue measure, let $H^{p}$ denote the usual Hardy space of functions on $\partial D$, and let $L_{a}^{p}$ denote the Bergman space of analytic functions on $D$ for which

$$
\|f\|_{L_{a}^{p}}=\left(\frac{1}{\pi} \iint_{D}|f(z)|^{p} d x d y\right)^{1 / p}<\infty .
$$

With $\phi \in L^{2}, S_{\phi}$ denotes the Hankel operator with symbol $\phi$, that is the operator with domain and range in $H^{2}$ and with matrix $\left(a_{i+j}\right)$, where the coefficient sequence $\left\{a_{n}\right\}$ is given by

$$
a_{n}=\hat{\phi}(-n) \quad(n \geq 0)
$$

In particular, when $\phi \in L^{\infty}$,

$$
S_{\phi}=P J M_{\phi} \mid H^{2},
$$

where $P$ is the orthogonal projection of $L^{2}$ onto $H^{2},(J f)(\zeta)=f(\bar{\zeta})\left(f \in L^{2}, \zeta \in \partial D\right)$, and $M_{\phi}$ is multiplication by $\phi$ (see Power [8]).

Several elementary functions will be needed, and it is convenient to list them here. With $w \in D, z \in D \cup \partial D$, write

$$
\begin{aligned}
& f_{w}(z)=(1-\bar{w} z)^{-3 / 2}, \quad v_{w}(z)=\left(1-|w|^{2}\right)^{1 / 2} /(1-\bar{w} z) \\
& g_{w}(z)=\left(1-|w|^{2}\right) /(1-\bar{w} z), \quad h_{w}(z)=z^{2} g_{w}(z) \\
& b_{w}(z)=2\left(1-|w|^{2}\right) /(1-\bar{w} z)^{3} .
\end{aligned}
$$

Glasgow Math. J. 28 (1986) 47-54. 
THEOREM 1. Let $g \in H^{2}$ and let $h(z)=z^{2} g(z)(z \in D)$, where $g(z)$ is the usual analytic extension of $g$ to $D$. Then $S_{\bar{g}}$ is trace class if and only if $h^{\prime \prime} \in L_{a}^{1}$. Also

$$
\frac{\pi}{8}\left\|h^{\prime \prime}\right\|_{L_{a}^{1}} \leq\left\|S_{\bar{g}}\right\|_{1} \leq\left\|h^{\prime \prime}\right\|_{L_{a}^{1}}
$$

The constant $\pi / 8$ is best possible.

Proof. Let $S_{\bar{g}}$ belong to the trace class. Then

$$
S_{\bar{s}}=\sum_{k=1}^{\infty} \lambda_{k}\left(u_{k} \otimes v_{k}\right)
$$

with $u_{k}, \dot{v}_{k} \in H^{2},\left\|u_{k}\right\|_{2}=\left\|v_{k}\right\|_{2}=1$ for all $k$ and with $\sum_{k=1}^{\infty}\left|\lambda_{k}\right|=\left\|S_{\bar{g}}\right\|_{1}$. For $w \in D$,

and so

$$
h^{\prime \prime}(w)=\frac{1}{\pi} \int_{0}^{2 \pi} g\left(e^{i \theta}\right) \frac{e^{3 i \theta}}{\left(e^{i \theta}-w\right)^{3}} d \theta
$$

Since

$$
\begin{aligned}
\overline{h^{\prime \prime}(w)} & =\frac{1}{\pi} \int_{0}^{2 \pi} \overline{g\left(e^{i \theta}\right)}\left(f_{w}\left(e^{i \theta}\right)\right)^{2} d \theta \\
& =2\left(\bar{g} f_{w}, \bar{f}_{w}\right) \\
& =2\left(\bar{g} f_{w}, J f_{\bar{w}}\right) \\
& =2\left(P J \bar{g} f_{w}, f_{\bar{w}}\right)=2\left(S_{\bar{g}} f_{w}, f_{\bar{w}}\right) .
\end{aligned}
$$

$$
\begin{aligned}
\left(S_{\bar{g}} f_{w}, f_{\bar{w}}\right) & =\sum_{k=1}^{\infty} \lambda_{k}\left(\left(u_{k} \otimes v_{k}\right) f_{w}, f_{\bar{w}}\right) \\
& =\sum_{k=1}^{\infty} \lambda_{k}\left(f_{w}, v_{k}\right)\left(u_{k}, f_{\bar{w}}\right), \\
h^{\prime \prime}(w) & =2 \sum_{k=1}^{\infty} \bar{\lambda}_{k}\left(v_{k}, f_{w}\right)\left(f_{\bar{w}}, u_{k}\right) .
\end{aligned}
$$

Given $v \in H^{2}$, let $(T v)(w)=\left(v, f_{w}\right)(w \in D)$. Then

with

$$
\begin{aligned}
(T v)(w) & =\frac{1}{2 \pi} \int_{0}^{2 \pi} v\left(e^{i \theta}\right)\left(1-w e^{-i \theta}\right)^{-3 / 2} d \theta \\
& =\sum_{n=0}^{\infty} \beta_{n} w^{n}
\end{aligned}
$$

$$
\beta_{n}=(-1)^{n}\left(\begin{array}{c}
-3 / 2 \\
n
\end{array}\right) \hat{v}(n)=\frac{1}{n !} \frac{3}{2} \cdot \frac{5}{2} \cdots \frac{(2 n+1)}{2} \hat{v}(n)
$$


Therefore

$$
\begin{aligned}
\|T v\|_{L_{a}^{2}}^{2} & =\frac{1}{\pi} \int_{0}^{1} \int_{0}^{2 \pi}\left|T v\left(r e^{i \theta}\right)\right|^{2} r d \theta d r \\
& =2 \int_{0}^{1} \sum_{n=0}^{\infty}\left|\beta_{n}\right|^{2} r^{2 n+1} d r=\sum_{n=0}^{\infty} \frac{\left|\beta_{n}\right|^{2}}{n+1} .
\end{aligned}
$$

Since $\alpha_{n}=\left(\begin{array}{c}-3 / 2 \\ n\end{array}\right)^{2} /(n+1)$ increases with $n$ and, by Stirling's formula, converges to $4 / \pi$, we have

$$
\|T v\|_{L_{a}^{2}}^{2} \leq \frac{4}{\pi}\|v\|_{2}^{2}
$$

Since also

$$
\left.\overline{\left(f_{\overline{1}}, u\right.}\right)=\left(u, f_{\bar{w}}\right)=(T u)(\bar{w}),
$$

the Cauchy-Schwarz inequality now gives

$$
\begin{aligned}
\left\|h^{\prime \prime}\right\|_{L_{a}^{1}} & \leq 2 \sum_{k=1}^{\infty}\left|\lambda_{k}\right|\left\|T v_{k}\right\|_{L_{a}^{2}}\left\|T u_{k}\right\|_{L_{a}^{2}} \\
& \leq \frac{8}{\pi} \sum_{k=1}^{\infty}\left|\lambda_{k}\right|\left\|v_{k}\right\|_{2}\left\|u_{k}\right\|_{2} \\
& =\frac{8}{\pi}\left\|S_{\bar{g}}\right\|_{1} .
\end{aligned}
$$

This proves that $h^{\prime \prime} \in L_{a}^{1}$ and establishes the first inequality. To complete the proof we need two lemmas. First however we note some properties of the elementary functions $g_{w}$, $h_{w}, b_{w}$.

A routine calculation gives

$$
S_{\bar{B}_{w}}=v_{\bar{w}} \otimes v_{w},
$$

and, since $\left\|v_{w}\right\|_{2}=1$, this shows that $S_{\bar{B}_{w}}$ is a rank one Hankel operator and

$$
\left\|S_{\bar{g}_{m}}\right\|_{1}=1 \quad(w \in D) \text {. }
$$

Since $h_{w}(z)=z^{2} g_{w}(z)$ and $h_{w}^{\prime \prime}=b_{w}$, inequality (2) shows that

$$
\left\|b_{w}\right\|_{L_{a}^{1}} \leq \frac{8}{\pi} \quad(w \in D) .
$$

Let $B$ denote the space of Bloch functions that vanish at 0 , that is functions $f$ analytic on $D$ with $f(0)=0$ and with $\left(1-|z|^{2}\right) f^{\prime}(z)$ bounded on $D$, and let

$$
\|f\|_{B}=\sup \left\{\left(1-|z|^{2}\right)\left|f^{\prime}(z)\right|: z \in D\right\} \text {. }
$$


Given $f \in L_{a}^{1}$ and $g \in B$, let

$$
\langle f, g\rangle=\frac{1}{\pi} \int_{0}^{1} \int_{0}^{2 \pi}\left(1-r^{2}\right) g^{\prime}\left(r e^{-i \theta}\right) f\left(r e^{i \theta}\right) r d \theta d r,
$$

which plainly satisfies

$$
|\langle f, g\rangle| \leq\|f\|_{L_{a}^{\prime}}\|g\|_{B} .
$$

It is known that $B$ represents the dual space of $L_{a}^{1}$ through $\langle$,$\rangle . (See [1], where a$ slightly different space is used in place of $L_{a}^{1}$.) In Lemma 2 we state this result with estimates for norms.

Let $c_{w}(z)=2(1-w z)^{-3}(w, z \in D)$, and given $\psi \in\left(L_{a}^{1}\right)^{*}$, let

$$
g_{\psi}(z)=\int_{[0, z]} \psi\left(c_{w}\right) d w
$$

where the integration is along the line segment joining 0 to $z$. Then $g_{\psi}$ is analytic in $D$ and

$$
\left(1-|z|^{2}\right) g_{\psi}^{\prime}(z)=\psi\left(b_{\bar{z}}\right) \quad(z \in D) .
$$

With (4), (6) shows that $g_{\psi} \in B$ and $\left\|g_{\psi}\right\|_{B} \leq \frac{8}{\pi}\|\psi\|$. Standard arguments now complete the proof of the following lemma.

Lemma 2. The mapping $\psi \rightarrow g_{\psi}$ is a linear bijection of $\left(L_{a}^{1}\right)^{*}$ onto $B$,

$$
\psi(f)=\left\langle f, g_{\psi}\right\rangle \quad\left(f \in L_{a}^{1}, \psi \in\left(L_{a}^{1}\right)^{*}\right),
$$

and

$$
\frac{\pi}{8}\left\|g_{\psi}\right\|_{B} \leq\|\psi\| \leq\left\|g_{\psi}\right\|_{B} \quad\left(\psi \in\left(L_{a}^{1}\right)^{*}\right)
$$

The following lemma is a modified version of the Coifman-Rochberg decomposition theorem [3] for $L_{a}^{1}$ with estimates of norms.

Lemma 3. $L_{a}^{1}$ is the set of functions $f$ of the form

$$
f=\sum_{k=1}^{\infty} \lambda_{k} b_{w_{k}}
$$

with $w_{k} \in D, \lambda_{k} \in \mathbb{C}$ and $\sum_{k=1}^{\infty}\left|\lambda_{k}\right|<\infty$. Also

$$
\frac{\pi}{8}\|f\|_{L_{a}^{1}} \leq \inf \sum_{k=1}^{\infty}\left|\lambda_{k}\right| \leq\|f\|_{L_{a}^{1}},
$$

where the infimum is taken over all decompositions (9) of $f$.

Proof. By Lemma 2 and (6),

$$
\|\psi\| \leq\left\|g_{\psi}\right\|_{B} \leq \sup \left\{\left|\psi\left(b_{z}\right)\right|: z \in D\right\} .
$$

Using also (4), the lemma now follows at once from ([2], Theorem 1). 
Proof of Theorem 1 continued. Suppose that $h^{\prime \prime} \in L_{a}^{1}$ and $\varepsilon>0$. By Lemma 3, there exist $w_{k} \in D, \lambda_{k} \in \mathbb{C}$ with $\sum_{k=1}^{\infty}\left|\lambda_{k}\right|<\left\|h^{\prime \prime}\right\|_{L_{a}^{1}}+\varepsilon$ and (since $h_{w}^{\prime \prime}=b_{w}$ ),

$$
h^{\prime \prime}=\sum_{k=1}^{\infty} \lambda_{k} h_{w_{k}}^{\prime \prime} \text {. }
$$

This series converges uniformly on compact subsets of $D$ since $\left|b_{w}(z)\right| \leq 2(1-|z|)^{-3}$, and so integration twice gives

$$
h=\sum_{k=1}^{\infty} \lambda_{k} h_{w_{k}}
$$

where we have used the fact that both sides have zero constant term and first degree term. Thus $g=\sum_{k=1}^{\infty} \lambda_{k} g_{w_{k}}, S_{\bar{g}}=\sum_{k=1}^{\infty} \bar{\lambda}_{k} S_{\bar{z}_{w_{k}}}$, and

$$
\left\|S_{\bar{g}}\right\|_{1} \leq \sum_{k=1}^{\infty}\left|\lambda_{k}\right|<\left\|h^{\prime \prime}\right\|_{L_{a}^{1}}+\varepsilon .
$$

It remains to prove that the constant $\pi / 8$ is best possible. Let $w \in D$ and $\phi_{w}(z)=\sqrt{2}\left(1-|w|^{2}\right)^{1 / 2} /(1-\bar{w} z)^{3 / 2}$, so that $b_{w}=\phi_{w}^{2}$. Then

$$
\left\|h_{w}^{\prime \prime}\right\|_{L_{a}^{1}}=\left\|\phi_{w}\right\|_{L_{a}^{2}}^{2}=2\left(1-|w|^{2}\right) \sum_{n=0}^{\infty} \alpha_{n}|w|^{2 n},
$$

with $\alpha_{n}=\left(\begin{array}{c}-3 / 2 \\ n\end{array}\right)^{2} /(n+1)$ as before. Since the sequence $\left\{\alpha_{n}\right\}$ increases, Abel's theorem gives

$$
\begin{aligned}
\sup _{w \in D}\left\|h_{w}^{\prime \prime}\right\|_{L_{a}^{1}} & =\sup _{0 \leq t<1} 2(1-t) \sum_{n=0}^{\infty} \alpha_{n} t^{n} \\
& =2 \lim _{t \rightarrow 1-0}\left\{\alpha_{0}+\sum_{n=1}^{\infty}\left(\alpha_{n}-\alpha_{n-1}\right) t^{n}\right\} \\
& =2\left\{\alpha_{0}+\sum_{n=1}^{\infty}\left(\alpha_{n}-\alpha_{n-1}\right)\right\} \\
& =2 \lim _{n \rightarrow \infty} \alpha_{n}=8 / \pi .
\end{aligned}
$$

Since $\left\|S_{\bar{g}_{w}}\right\|_{1}=1$, this completes the proof of the theorem.

Remarks. (1) We have proved that

$$
\left\|S_{\bar{g}}\right\|_{1} \leq C\left\|h^{\prime \prime}\right\|_{L_{a}^{1}} \quad\left(h^{\prime \prime} \in L_{a}^{1}\right),
$$

for some constant $C \leq 1$, but we do not know whether 1 is the best value of $C$. It is easy to prove that $C \geq \frac{1}{2}$, by taking $g=g_{0}(=1)$. We have $\left\|S_{\bar{g}_{0}}\right\|=1$ and $\left\|h_{0}^{\prime \prime}\right\|_{L_{a}^{1}}=2$.

(2) The same constants $\pi / 8$ and 1 occur also in Lemmas 2 and 3 and it is natural to ask whether they are best possible.

We have already proved in (11) that

$$
\sup _{w \in D}\left\|b_{w}\right\|_{L_{a}^{1}}=8 / \pi
$$


This shows at once that the constant $\pi / 8$ is best possible in Lemma 3, for with $f=b_{w}$ we have inf $\sum_{k=1}^{\infty}\left|\lambda_{k}\right| \leq 1$. It follows that $\pi / 8$ is also best possible in Lemma 2 . For suppose that $C>0$ with

$$
C\left\|g_{\psi}\right\|_{B} \leq\|\psi\| \quad\left(\psi \in\left(L_{a}^{1}\right)^{*}\right) .
$$

Given $f \in L_{a}^{1}$, there exists $\psi \in\left(L_{a}^{1}\right)^{*}$ with $\|\psi\|=1$ and $\psi(f)=\|f\|_{L_{a}^{1}}$. If $f=\sum_{k=1}^{\infty} \lambda_{k} b_{w^{\prime} k}$, it follows that

$$
\begin{aligned}
\|f\|_{L_{a}^{1}} & \leq \sum_{k=1}^{\infty}\left|\lambda_{k}\right|\left|\psi\left(b_{w w_{k}}\right)\right| \\
& \leq \sum_{k=1}^{\infty}\left|\lambda_{k}\right| \sup _{w \in D}\left|\psi\left(b_{w}\right)\right| \\
& \leq \sum_{k=1}^{\infty}\left|\lambda_{k}\right|\left\|g_{\psi}\right\|_{B} \leq C^{-1} \sum_{k=1}^{\infty}\left|\lambda_{k}\right| .
\end{aligned}
$$

This gives $C\|f\|_{L_{a}^{1}} \leq \inf \sum_{k=1}^{\infty}\left|\lambda_{k}\right|$, and so $C \leq \pi / 8$ by the result just proved for Lemma 3 .

Next we prove that if $\|\psi\| \leq C\left\|g_{v}\right\|_{B}$ for all $\psi \in\left(L_{n}^{1}\right)^{*}$, then $C \geq e / 4$. For this we take $f(z)=z^{n-1}, g(z)=z^{n}$. Then $\|f\|_{L_{a}^{1}}=2 /(n+1),\|g\|_{B}=\frac{2 n}{n+1}\left(1-\frac{2}{n+1}\right)^{\frac{n-1}{2}}$ so that $\lim _{n \rightarrow \infty}\|g\|_{B}=2 / e$, while $\langle f, g\rangle=1 /(n+1)$.

It now follows also that if inf $\sum_{k=1}^{\infty}\left|\lambda_{k}\right| \leq C\|f\|_{L_{a}^{1}}$ for all $f \in L_{a}^{1}$, then $C \geq e / 4$. For given $f \in L_{a}^{1}$ with $\|f\|_{L_{a}^{1}}=1$ and $\varepsilon>0$, we choose $w_{k}, \lambda_{k}$ with $f=\sum_{k=1}^{\infty} \lambda_{k} b_{w_{k}}$ and $\sum_{k=1}^{\infty}\left|\lambda_{k}\right|<C+\varepsilon$. Given $\psi \in\left(L_{a}^{1}\right)^{*}$, we have

$$
\begin{gathered}
|\psi(f)|=\left|\sum_{k=1}^{\infty} \lambda_{k} \psi\left(b_{w_{k}}\right)\right| \leq(C+\varepsilon)\left\|g_{\psi}\right\|_{B}, \\
\|\psi\| \leq C\left\|g_{\psi}\right\|_{B},
\end{gathered}
$$

and so $C \geq e / 4$ by the corresponding result for Lemma 2 .

A Hankel operator valued projection on the trace class. Let $\mathscr{C}_{1}$ denote the Banach space of trace class operators on $H^{2}, \mathscr{S} \cap \mathscr{C}_{1}$ the closed subspace of Hankel operators in $\mathscr{C}_{1}$. As a corollary of Theorem 1 we obtain a bounded projection $P_{\mathscr{S}}$ on the space $\mathscr{C}_{1}$ with range $\mathscr{S} \cap \mathscr{C}_{1}$, together with a satisfactory estimate of its norm. $P_{\mathscr{S}}$ is the special case $P_{1 / 2,1 / 2}$ of a family of projections $P_{\alpha, \beta}$ of this kind found by A. B. Aleksandrov, and it is known that the natural averaging projection is not bounded on $\mathscr{C}_{1}$, though it is bounded on the Schatten-von Neumann spaces with $1<p<\infty$. (See Peller $[4,6,7]$ ).

Given an operator $A \in \mathscr{C}_{1}$ with matrix $\left(a_{i j}\right)$ relative to the natural basis, we define $P_{\mathscr{S}} A$ to be the Hankel operator with coefficient sequence $\left\{b_{n}\right\}$ given by

$$
b_{n}=\frac{2(-1)^{n}}{(n+2)(n+1)} \sum_{i+j=n}\left(\begin{array}{c}
-3 / 2 \\
i
\end{array}\right)\left(\begin{array}{c}
-3 / 2 \\
j
\end{array}\right) a_{i j}
$$


Comparison of the coefficient of $z^{n}$ in the expansions of $\left((1-z)^{-3 / 2}\right)^{2}$ and $(1-z)^{-3}$ shows that $b_{n}=c_{n}$ if $a_{i j}=c_{i+j}$, and so $P_{S P}$ is a projection.

COROLlary 4 . The projection $P_{\mathscr{S}}$ defined by $(12)$ is a bounded projection on $\mathscr{C}_{1}$ with range $\mathscr{C}_{1} \cap \mathscr{Y}$, and

$$
\left\|P_{\mathscr{S P}}\right\| \leq 8 / \pi \text {. }
$$

Proof. Let $A=u \otimes v$ with $u, v \in H^{2}$. The matrix $\left(a_{i j}\right)$ of $A$ is given by $a_{i j}=\hat{u}_{i} \hat{v}_{j}$, where $\hat{u}_{n}, \hat{v}_{n}$ are the Fourier coefficients of $u, v$. Therefore the coefficient sequence $\left\{b_{n}\right\}$ of the Hankel operator $P_{\mathscr{S}} A$ is given by

$$
b_{n}=\frac{2(-1)^{n}}{(n+2)(n+1)} \sum_{i+j=n}\left(\begin{array}{c}
-3 / 2 \\
i
\end{array}\right)\left(\begin{array}{c}
-3 / 2 \\
j
\end{array}\right) \hat{u}_{i} \overline{\hat{v}}_{j}
$$

Let $g(z)=\sum_{n=0}^{\infty} b_{n} z^{n}, h(z)=z^{2} g(z)$. Then

with

$$
h^{\prime \prime}(z)=\sum_{n=0}^{\infty}(n+2)(n+1) b_{n} z^{n}=2 \psi_{1}(z) \psi_{2}(z)
$$

$$
\psi_{1}(z)=\sum_{k=0}^{\infty}(-1)^{k}\left(\begin{array}{c}
-3 / 2 \\
k
\end{array}\right) \hat{u}_{k} z^{k}, \quad \psi_{2}(z)=\sum_{k=0}^{\infty}(-1)^{k}\left(\begin{array}{c}
-3 / 2 \\
k
\end{array}\right) \overline{\hat{v}}_{k} z^{k}
$$

By Theorem 1,

$$
\left\|P_{S_{S}} A\right\|_{1}=\left\|S_{\bar{g}}\right\|_{1} \leq\left\|h^{\prime \prime}\right\|_{L_{a}^{1}} \leq 2\left\|\psi_{1}\right\|_{L_{a}^{2}}\left\|\psi_{2}\right\|_{L_{a}^{2}}
$$

By a calculation in the proof of Theorem $1,\left\|\psi_{1}\right\|_{L_{a}^{2}} \leqslant\left(\frac{4}{\pi}\right)^{1 / 2}\|u\|_{2}$, and similarly for $\left\|\psi_{2}\right\|_{L_{a}^{2}}$ Thus

$$
\left\|P_{\mathscr{S P}} A\right\|_{1} \leq 8 / \pi\|u\|_{2}\|v\|_{2}=8 / \pi\|A\|_{1}
$$

Given arbitrary $A \in \mathscr{C}_{1}$, we have $A=\sum_{k=1}^{\infty} \lambda_{k} A_{k}$, with $A_{k}=u_{k} \otimes v_{k},\left\|A_{k}\right\|_{1}=1$, and $\sum_{k=1}^{\infty}\left|\lambda_{k}\right|=\|A\|_{1}$. The series $\sum_{k=1}^{\infty} \lambda_{k} P_{S \rho} A_{k}$ converges in the Banach space $\mathscr{C}_{1}$ to a Hankel operator $S$ and $\|S\|_{1} \leq \frac{8}{\pi} \sum_{k=1}^{\infty}\left|\lambda_{k}\right|=\frac{8}{\pi}\|A\|_{1}$. If $\left\{b_{n}^{(k)}\right\}$ is the coefficient sequence of $P_{s g} A_{k}$, then the coefficient sequence of $S$ is $\left\{b_{n}\right\}$ given by

$$
b_{n}=\sum_{k=1}^{\infty} \lambda_{k} b_{n}^{(k)}=\sum_{k=1}^{\infty} \lambda_{k} \frac{2(-1)^{n}}{(n+2)(n+1)} \sum_{i+j=n}\left(\begin{array}{c}
-3 / 2 \\
i
\end{array}\right)\left(\begin{array}{c}
-3 / 2 \\
j
\end{array}\right) a_{i j}^{(k)}
$$
where $\left(a_{i j}^{(k)}\right)$ is the matrix of $A_{k}$. Since the matrix $\left(a_{i j}\right)$ of $A$ is given by $a_{i j}=\sum_{k=1}^{\infty} \lambda_{k} a_{i j}^{(k)}$, it
follows that

$$
b_{n}=\frac{2(-1)^{n}}{(n+2)(n+1)} \sum_{i+j=n}\left(\begin{array}{c}
-3 / 2 \\
i
\end{array}\right)\left(\begin{array}{c}
-3 / 2 \\
j
\end{array}\right) a_{i j}
$$

and we have proved that $S=P_{\mathscr{S}} A$. 
Remark. The proof of Corollary 4 has used the inequality $\left\|S_{\bar{g}}\right\|_{1} \leq\left\|h^{\prime \prime}\right\|_{L_{a}^{1}}$ from Theorem 1, which we do not know to be best possible. If this can be improved to $\left\|S_{\bar{g}}\right\|_{1} \leq c\left\|h^{\prime \prime}\right\|_{L_{a}^{1}}$ for some $c<1$, we obtain the improved estimate $\left\|P_{\mathscr{S}}\right\| \leq c 8 / \pi$ for the norm of $P_{\mathscr{S}}$.

\section{REFERENCES}

1. J. M. Anderson, J. Clunie and $\mathrm{Ch}$. Pommerenke, On Bloch functions and normal functions, J. Reine Angew. Math. 270 (1974), 12-37.

2. F. F. Bonsall, Decompositions of functions as sums of elementary function, Quart. J. Math. (Oxford), to appear.

3. R. R. Coifman and R. Rochberg, Representation theorems for holomorphic and harmonic functions in $L^{p}$, Asterisque 77 (1980), 11-66.

4. V. V. Peller, Hankel operators of class $\varsigma_{p}$ and their applications (Rational approximation, Gaussian processes, the problem of majorizing operators), Mat. Sbornik 113 (1980) No. 4, 538-581 (Russian). English translation: Math. USSR Sbornik 41 (1982), No. 4, 443-479.

5. V. V. Peller, Nuclearity of Hankel operators Preprint, Leningrad (1979).

6. V. V. Peller, Continuity of the averaging projection onto the set of Hankel matrices, Preprint, Leningrad (1983).

7. V. V. Peller, Continuity of the averaging projection onto the set of Hankel matrices II, Preprint, Leningrad (1983). 1982).

8. S. C. Power, Hankel operators on Hilbert space, Research notes in mathematics 64 (Pitman,

Departmant of Mathematics

UNIVERSITY OF EDINBURGH
Department of Mathematics, Maynooth College, Co. KILDARE 